\title{
Validation of a Fast Block-Iterative Spatio-temporal Reconstruction Algorithm for Small Animal Dynamic PET Data
}

\author{
Tatsuya Kon, ${ }^{\#, ~}$ Takashi OBI, ${ }^{* *}$ Muneyuki Sakata, ${ }^{* * *}$ Nagaaki Ohy ama, $^{* *}$ Yuichi Kimura $^{\dagger}$
}

\begin{abstract}
Spatio-temporal reconstruction methods for positron emission tomography estimate the tissue timeactivity curves that are required for functional imaging. Since the time-related change of activity at a voxel has a temporal correlation by itself, temporal basis function approaches can be adopted. However, these image reconstruction methods suffer from a high computational cost. We have proposed a novel spatio-temporal reconstruction method using a temporal basis function approach, which is based on a fast block-iterative algorithm named Dynamic Row-Action Maximum-Likelihood Algorithm (DRAMA). Using the proposed method, data quickly converge to an estimate after around two iterations. This study aimed to validate the performance of the proposed algorithm on small animal PET data. We applied the proposed method to ${ }^{18} \mathrm{~F}$-FDG mouse dynamic PET data. A parametric image of regional glucose metabolism reconstructed from the proposed method was almost identical to those obtained from conventional reconstruction algorithms. The proposed method took $14.3 \mathrm{~h}$ for computation, which was twice as fast as conventional algorithms. These results support the usability of the proposed algorithm for voxel-by-voxel estimation.
\end{abstract}

Keywords : PET, parametric image, spatio-temporal reconstruction, DRAMA.

Adv Biomed Eng. 3: pp. 7-13, 2014.

\section{Introduction}

Positron emission tomography (PET) allows imaging of various functionalities of living tissues. For detailed functional imaging, in order to take into account the behavior of the administered radiopharmaceutical in a target tissue, the time history of radioactivity concentrations in a tissue and arterial plasma are needed. This process is called a kinetic study [1]. If a kinetic study is conducted for each voxel, the derived images are called parametric images, and these images provide the functionality. Parametric images are valuable for quantifying physiological and biochemical information, which can clarify disease mechanisms or vital functions in human or animal bodies. The parameter values of the images can be

This study was presented at the Symposium on Biomedical Engineering 2013, Fukuoka, September, 2013.

Received on July 28, 2013; revised on November 18, 2013; accepted on January 21, 2014.

* Interdisciplinary Graduate School of Science and Engineering, Tokyo Institute of Technology, Kanagawa, Japan.

** Imaging Science and Engineering Laboratory, Tokyo Institute of Technology, Kanagawa, Japan.

*** Research Team for Neuroimaging, Tokyo Metropolitan Institute of Gerontology, Tokyo, Japan.

$\dagger$ Faculty of Biology-Oriented Science and Technology, Kinki University, Wakayama, Japan.

\# 4259-R2-60 Nagatsuta-cho, Midori-ku, Yokohama, Kanagawa 226-8503, Japan.

E-mail:kon.t.aa@m.titech.ac.jp estimated voxel-by-voxel from a plasma time-activity curve (pTAC) and tissue time-activity curves (tTACs), using a kinetic model analysis such as the Patlak graphic plot method [2]. The tTACs indicate the temporal changes of tracer activity, and can be obtained from timesequential PET images.

A general approach to acquiring temporal sequences of PET images is frame-by-frame reconstruction. In this approach, each frame is reconstructed independently, and the temporal relation is not taken into account. This approach may deteriorate the image quality.

To take into account the temporal correlation within each tTAC, novel spatio-temporal reconstruction methods have been developed, in which tTACs are modeled using temporal basis functions. The use of cubic B-spline basis functions was first reported[3]. Further study estimated basis functions from acquired PET data[4]. These studies showed improvement of image quality, but both algorithms had the disadvantage of high computational cost required to maximize the likelihood function.

The computational cost for image reconstruction has been investigated. In terms of spatial image reconstruction from static PET data, block-iterative algorithms have been reported to reduce the computational demand of maximum-likelihood (ML) estimation. Row-action maximum likelihood algorithm (RAMLA) [5] reduced the number of iterations by introducing a relaxation parameter, and successfully accelerated the reconstruction process. In dynamic RAMLA (DRAMA) [6], the relaxation parameter of RAMLA was modified to maintain the signal-to-noise ratio among iterations. Such blockgradient methods for list-mode data (a list of detection 
information of the photon events) were derived by Nakayama and Kudo [7], who proposed a method to decompose the list-mode likelihood function into a set of partial functions.

Our previous study presented a derivation of DRAMA applied to spatio-temporal reconstruction with temporal basis functions $[8,9]$. When evaluated using digital phantom data, the data converged quickly. Moreover, the performance of the algorithm with various parameter definitions was investigated [9].

The purpose of the present study was to validate the performance of the proposed method for real PET data. We conducted estimation using ${ }^{18} \mathrm{~F}$-FDG mouse data. The results show that the proposed method converges more quickly than conventional methods.

\section{Methods}

\subsection{Algorithm}

In PET scanning, pairs of photons arising from positron emissions are detected by external detectors. A list-mode data format is a format in which the detected information, including detector pair indices and detection time, is recorded event-by-event.

We let $S$ be the list-mode data set, and assume the $n$ th photon event has detection information; detector pair $i_{n}$ and detection time $t_{n}$ (Fig. 1). Let $Y_{i}$ be the set of listmode data detected at a particular detector pair $i$, i.e.,

$$
Y_{i}=\left\{t_{n} \mid i_{n}=i\right\} \text {. }
$$

The component $Y_{i}$ depends on the tTACs denoted by $x_{j}(t)$, where $j$ is the voxel index $\left(1 \leq J \leq N_{j}\right)$ and $N_{j}$ is the number of voxels. The expected amounts of $Y_{i}$ can be represented by a temporal function $\bar{y}_{i}(t)$ that satisfies

$$
\bar{y}_{i}(t)=\sum_{j=1}^{N_{j}} a_{i j} x_{j}(t)
$$

where $a_{i j}$ denotes the probability that a photon emitted from voxel $j$ will be detected by pair $i$ (Fig. 2).

The probability of the detection of $Y_{i}$ is distributed as a Poisson process because $S$ originates from the radiation process. Consequently, according to [2], it can be represented by

$$
\mathrm{P}\left(Y_{i} \mid\left\{x_{j}(t)\right\}\right)=\left(\bar{y}_{i}\left(t_{n}\right)\right) \exp \left(-\int_{T_{0}}^{T_{1}} \bar{y}_{i}(t) d t\right)
$$

where $T_{0}$ denotes the start and $T_{1}$ the end time of a scan.

We assume that $x_{j}(t)$ can be represented by a linear combination of a temporal basis function set[3] given by

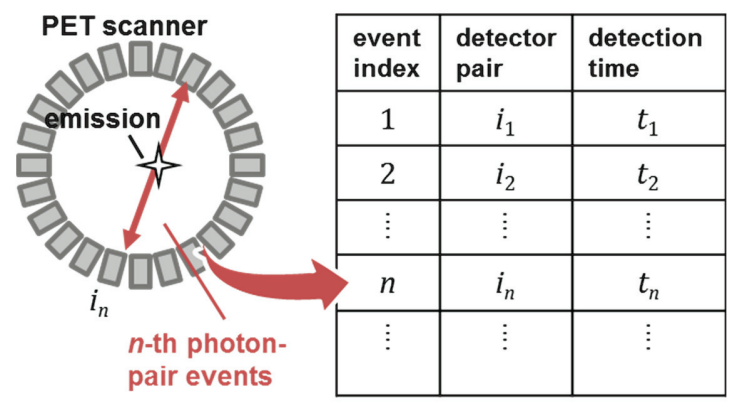

Fig. 1 Conceptual image of a list-mode data format.

$$
x_{j}(t)=\sum_{l=1}^{N_{l}} w_{j l} B_{l}(t)
$$

where $B_{l}(t)$ is the $l$-th basis function $\left(1 \leq l \leq N_{l}\right)$, and $w_{j l}$ is the basis function coefficient. Substituting ( 4 ) into ( 2 ) and considering the probability of all the list-mode data, we obtain a list-mode log-likelihood function

$$
\begin{aligned}
L\left(\left\{w_{j l}\right\}\right)= & \sum_{n \in S}\left\{\ln \left(\sum_{j=1}^{N_{j}} a_{i j} \sum_{l=1}^{N_{l}} w_{j l} B_{l}\left(t_{n}\right)\right)\right\} \\
& -\sum_{i=1}^{N_{i}}\left\{a_{i j} \sum_{l=1}^{N_{l}} \int_{T_{0}}^{T_{1}} w_{j l} B_{l}(t) d t\right\},
\end{aligned}
$$

where $N_{i}$ is the number of detector pairs. The optimal $\left\{w_{j l}\right\}$, which maximizes $L$, gives estimated tTACs. According to [3], a penalized log-likelihood function can also be used instead of ( 5 ) to take into account some prior information such as a neighborhood or non-negative penalty.

In our previous study [8], the 4D-DRAMA method was derived to maximize partial functions $L_{m}$, where we divided the list-mode data into $M$ subsets denoted by $S_{m}$ $(0 \leq m \leq M-1)$ and divided the log-likelihood function into $M$ partial functions. The introduction of subsets allows us to update the estimation $M$ times within a main iteration ; thus, the convergence speed can be accelerated. The partial functions $L_{m}$ and subsets $S_{m}$ satisfy

$$
\begin{aligned}
& L\left(\left\{w_{j l}\right\}\right)=\sum_{m=0}^{M-1} L_{m}\left(\left\{w_{j l}\right\}\right), \\
& S=S_{0}+S_{1}+\ldots+S_{M-1}=\sum_{m=0}^{M-1} S_{m} .
\end{aligned}
$$

The update equations are defined so that $w_{j l}$ maximizes the partial function $(6)$,

$$
\begin{aligned}
& w_{j l}^{(k, 0)}=w_{j l}^{(k)}, w_{j l}^{(k+1)}=w_{j l}^{(k, M)}, \\
& w_{j l}^{(k, m+1)}=w_{j l}^{(k, m)}+\lambda^{(k, m)} \frac{w_{j l}^{(k, m)}}{C_{j l}} \sum_{n \in S_{m}} a_{i_{n} j} B_{l}\left(t_{n}\right) . \\
& \left(\frac{1}{\sum_{j^{\prime}} a_{i_{n} j^{\prime}} \sum_{l^{\prime}} w_{j l^{\prime}}^{(k, m)} B_{l^{\prime}}(t)}-p_{m j l}\right),
\end{aligned}
$$

where $w_{j l}^{(k, m)}$ is the estimated coefficient after the $k$-th iterations and $m$-th sub-iterations. The $p_{m j l}$ in $(9)$ is called a blocking factor, and is a constant value used to decompose the log-likelihood into partial functions. In this paper we use the definition reported previously [9]

$$
p_{m j l}=\frac{\sum_{i} a_{i j} \int_{T_{0}}^{T_{1}} B_{l}(t) d t}{\sum_{m^{\prime}} \sum_{n \in S_{m^{\prime}}} a_{i_{n} j} B_{l}\left(t_{n}\right)} .
$$

The normalization coefficient $C_{j l}$ is represented by

$$
C_{j l}=\max _{m} \sum_{n \in S_{m}} p_{m j l} a_{i_{n}} B_{l}\left(t_{n}\right),
$$

and $\lambda^{(k, m)}$ is a relaxation parameter expressed as

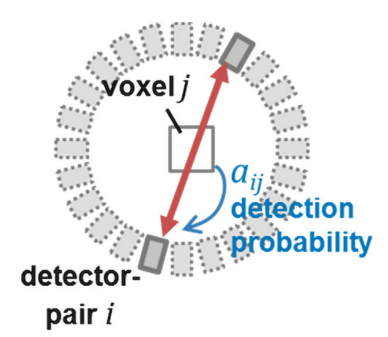

Fig. 2 Conceptual image of detection probability between a detector pair and a voxel. 


$$
\lambda^{(k, m)}=\frac{\beta_{0}}{\beta_{0}+m+\gamma k M},
$$

with constant values $\gamma$ (defined empirically) and $\beta_{0}$ (calculated using geometrical correlation information).

\subsection{Experiment}

Our experiment used ${ }^{18}$ F-FDG mouse dynamic PET data acquired on an Inveon Dedicated PET scanner (Siemens Medical Solutions Inc., Malvern, PA, USA). Three mice were tested. Each mouse was injected an average of 9.27 $\mathrm{MBq}$ of ${ }^{18} \mathrm{~F}$-FDG solution in $100 \mu \mathrm{L}$ of saline over $30 \mathrm{~s}$. The total acquisition time was $60 \mathrm{~min}$. The data consisted of $1.09 \times 10^{8}$ counts on average.

We assumed the effects of attenuation and scatter coincidences to be negligible because of the sufficiently small size of the mice. We also neglected the random coincidences since the number of delay coincidence events was about $2.1 \%$ of the number of prompt events. A PE-10 catheter was inserted into the femoral artery, and micro-liter order arterial blood was sampled serially using a CD-well [10] to measure pTAC. The protocol was validated by the Institutional Animal Care and Use Committee at the National Institute of Radiological Sciences (Chiba, Japan), and the mice were handled in accordance with the recommendations of the US National Institutes of Health. We compared the performance of the proposed method with conventional methods using 4DMLEM (MLEM with temporal basis functions) and frameby-frame 3D-MLEM. Reconstructed image voxels measured $0.1 \mathrm{~cm}$ each side. Detection sensitivities were calculated from detector position information, since the values are unpublished. Thus we failed to make exact comparison between a scanner bundled reconstruction method and the list-mode based methods. However, as reference, we have included the tTACs reconstructed by bundled filtered back projection (FBP) method with voxel size of $0.03 \mathrm{~cm}$. In 4D-DRAMA, the relaxation parameters were set to be $\beta_{0}=57.3$ and $\gamma=0.1[8]$. As temporal basis functions, we selected a set of cubic B-spline basis functions (the third-fold convoluted value of the rectangular pulse functions), the knot vector (which determines the curve forms) of which was log-spaced from $10 \mathrm{sec}$ to the last second (as shown in Fig. 3). The list-mode data were divided into $M=10$ subsets. The same basis function set was used for both the proposed method and 4D-MLEM. Frame-by-frame 3D-MLEM was calculated for each of the 25 time frames $(6$ of $10 \mathrm{~s}, 4$ of $15 \mathrm{~s}, 5$ of 1 min, 4 of $2 \mathrm{~min}, 3$ of $5 \mathrm{~min}$, and 3 of $10 \mathrm{~min}$ ) over a 60 -min scan. The reconstruction images were filtered by a Gaussian spatial filter with $0.2 \mathrm{~cm}$ full width at half maximum.

We recorded the normalized mean square difference of updated estimates (NMS) within a cardiac region of interest (ROI). We defined NMS as

$$
\mathrm{NMS}=\frac{1}{R} \sum_{r} \frac{\left(f_{r}^{(k+1)}-f_{r}^{(k)}\right)^{2}}{\left(f_{r}^{(k)}\right)^{2}},
$$

where $f_{r}$ is the updated estimate $\left(w_{j l}\right.$ for $4 \mathrm{D}$-estimations or voxel value of a frame for frame-by-frame EM) within the ROI, and $R$ is the number of estimates. Then, we considered that the image values had converged when the

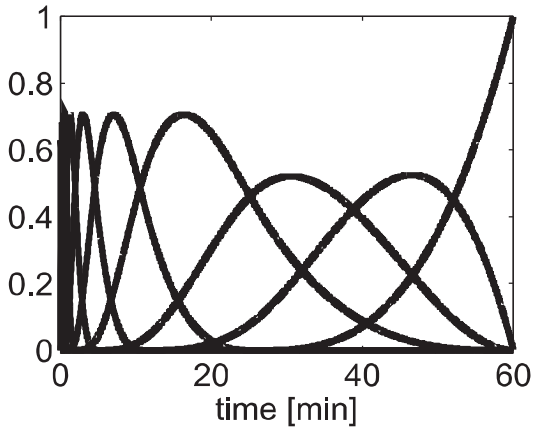

Fig. 3 Cubic B-spline basis functions for $4 \mathrm{D}$ reconstruction methods.
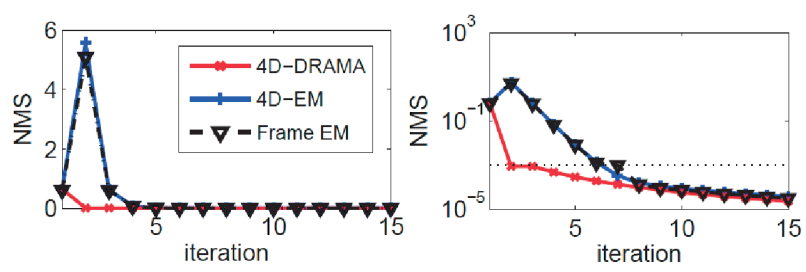

Fig. 4 Normalized mean square of update estimates through 15 iterations for Mouse 1, using different reconstruction methods[linear (left) and semi-log (right) plots]

Table 1 Mean iterations needed for convergence and computational time for reconstruction processes of three mice.

\begin{tabular}{ccccc}
\hline \multirow{2}{*}{ algorithm } & \multirow{2}{*}{ iteration } & \multicolumn{3}{c}{ computational time[h] } \\
& & mean & $\max$ & $\min$ \\
\hline 4D-DRAMA & 2.7 & 14.3 & 18.8 & 9.48 \\
4D-EM & 7.0 & 26.2 & 31.5 & 22.2 \\
Frame EM & 7.3 & 31.5 & 35.1 & 27.5 \\
\hline
\end{tabular}

NMS became sufficiently small $(<0.001)$.

From the reconstructed tTACs, we produced parametric images by means of the Patlak graphic plot analysis [1] that generates parametric images of ${ }^{18} \mathrm{~F}-\mathrm{FDG}$ influx values $K_{i}$. This process was applied to the tTACs reconstructed by each of the three different methods.

All reconstruction processes were conducted on an Intel Xeon X5670 $(2.93 \mathrm{GHz})$ with 12 core threads and 24 GB memory and were implemented in $\mathrm{C}++$. The $\mathrm{ROI}$ with 21 voxels was placed in a cardiac region.

\section{Results}

Figure 4 shows the normalized mean square of the updated values of the ROI through 15 iterations for Mouse 1. The values became less than 0.001 after two iterations in 4D-DRAMA, after seven iterations in 4D-MLEM method, and after eight iterations in frame-by-frame 3DMLEM.

Table 1 shows the mean number of iterations and computational time required for reconstruction in three mice. The mean computational time of 4D-DRAMA was 
1.8 and 2.2 times shorter than 4D-MLEM and frame-byframe EM, respectively. The computational time included pre-computation of the blocking factor $p_{m j l}$ for $4 \mathrm{D}$ DRAMA. As an example, the computation time for a mouse dataset (Mouse 1) using 4D-DRAMA comprised $2.33 \mathrm{~h}$ for pre-computation and $3.6 \mathrm{~h}$ per iteration, while that using 4D-MLEM comprised $3.2 \mathrm{~h}$ per iteration, and that using frame-by-frame EM comprised $3.7 \mathrm{~h}$ per
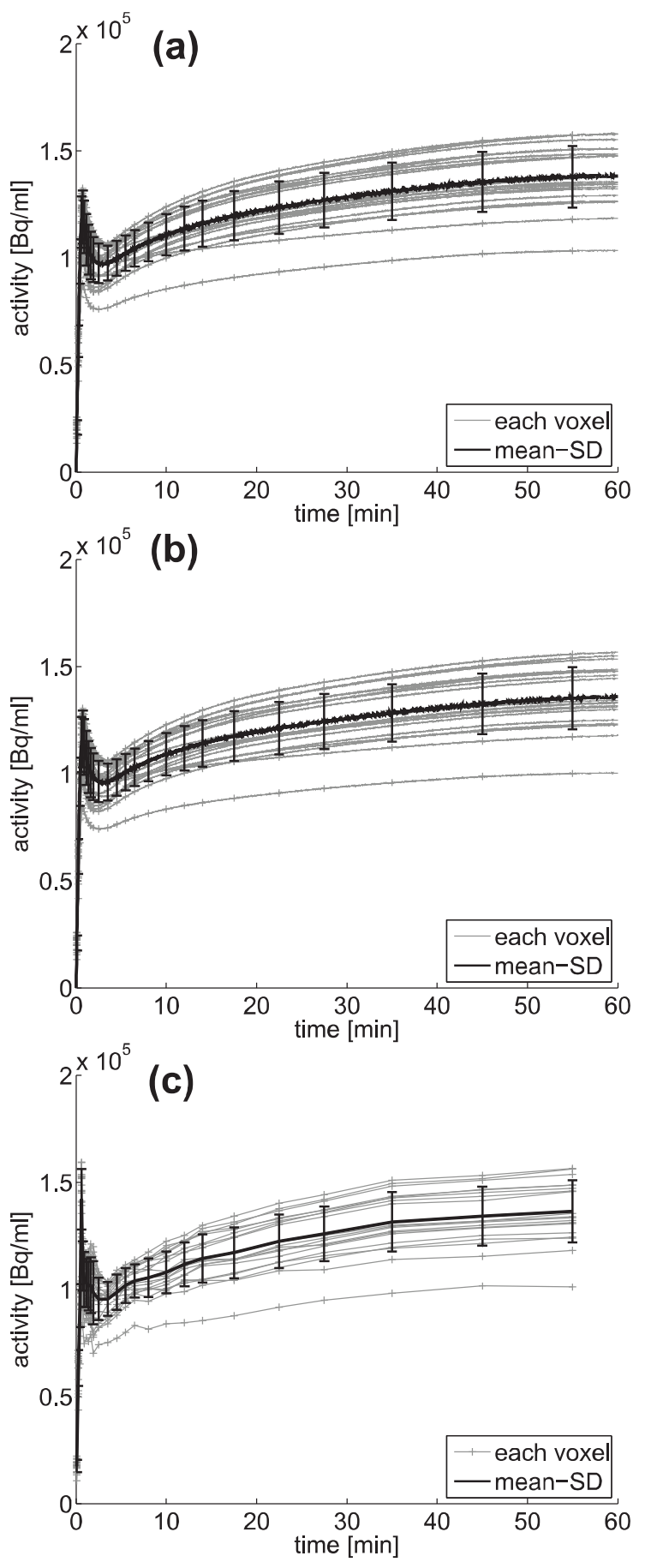

Fig. 5 Reconstructed tTACs in ROI of Mouse 1 (over 60 min of scan time). The curves were calculated using ( a ) 4D-DRAMA, ( b ) 4D-MLEM, and ( c ) frame-byframe EM. Gray lines depict the curves of each voxel within the ROI, and black lines depict the mean and standard deviation (SD). iteration.

The estimated tTACs in the ROI are shown in Fig. 5, and the early phase curves are depicted in Fig. 6. The black lines indicate the mean curves and standard deviations $(\mathrm{SD})$, and the gray lines indicate voxel-wise tTACs. In addition, the mean tTACs in the ROI are plotted in Fig. 7. In this figure, we also plotted the mean curve of FBP images (within much the same ROI of 172 pixels) as reference; this curve was obtained by the
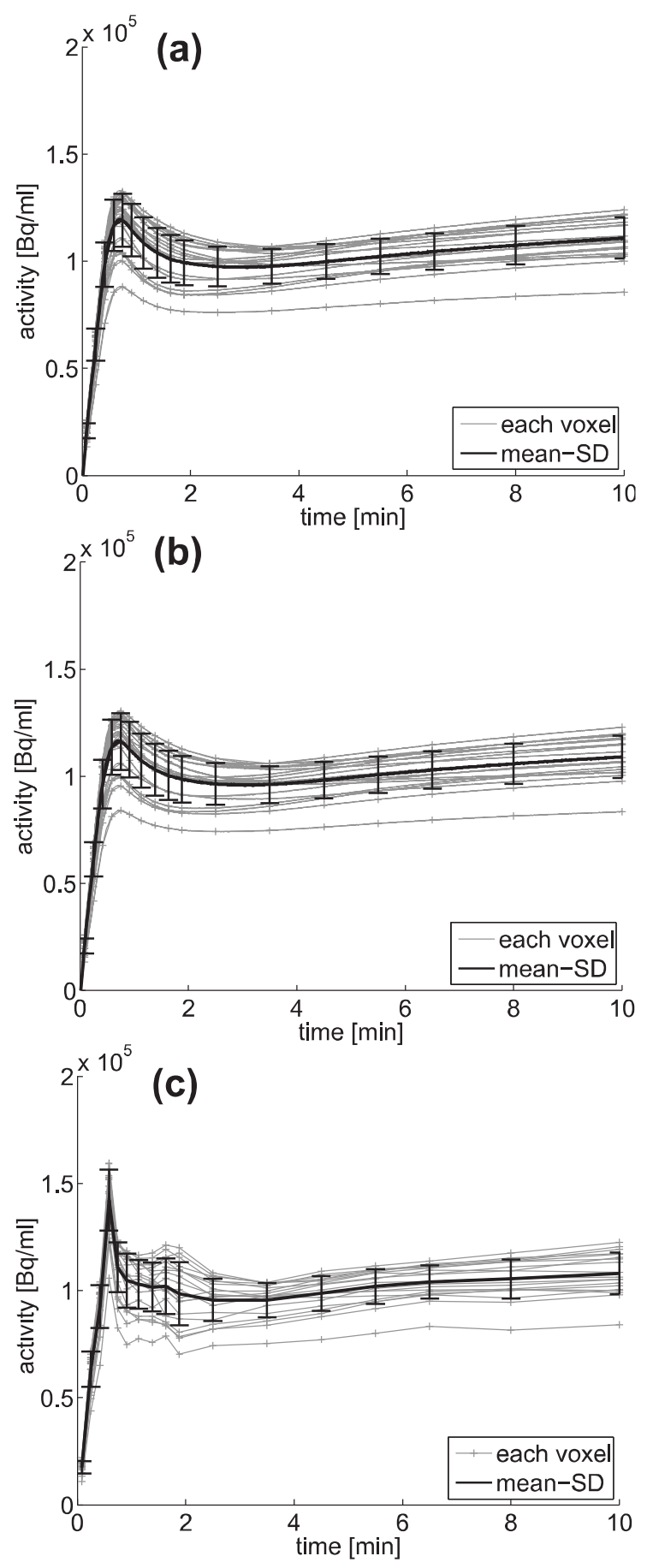

Fig. 6 Reconstructed tTACs in ROI of Mouse 1 (for the first $10 \mathrm{~min}$ of scan time). The curves were calculated using ( a ) 4D-DRAMA, ( b ) 4D-MLEM, and ( c ) frame-by-frame EM. The lines depict data as in Fig. 5. 
Table 2 ROI evaluation of ${ }^{18}$ F-FDG influx images.

\begin{tabular}{cccccccccc}
\hline & \multicolumn{3}{c}{ Mouse 1 } & \multicolumn{3}{c}{ Mouse 2} & \multicolumn{3}{c}{ Mouse 3 } \\
\cline { 2 - 9 } algorithm & $\begin{array}{c}\text { mean } \\
{\left[\times 10^{-2}\right]}\end{array}$ & $\begin{array}{c}\text { SD } \\
{\left[\times 10^{-3}\right]}\end{array}$ & $p$ value & $\begin{array}{c}\text { mean } \\
{\left[\times 10^{-2}\right]}\end{array}$ & $\begin{array}{c}\text { SD } \\
{\left[\times 10^{-3}\right]}\end{array}$ & $p$ value & $\begin{array}{c}\text { mean } \\
{\left[\times 10^{-2}\right]}\end{array}$ & $\begin{array}{c}\text { SD } \\
{\left[\times 10^{-3}\right]}\end{array}$ & $p$ value \\
\hline 4D-DRAMA & 5.08 & 4.03 & - & 11.6 & 18.5 & - & 2.38 & 2.14 & - \\
4D-EM & 5.00 & 5.58 & 0.19 & 11.3 & 17.1 & 0.057 & 2.35 & 2.18 & 0.069 \\
Frame EM & 5.10 & 5.53 & 0.68 & 11.5 & 17.4 & 0.22 & 2.38 & 2.18 & 0.30 \\
\hline
\end{tabular}

The mean and SD values were calculated using the influx values of mouse data within the ROI, and $p$ values were obtained from paired t-tests comparing 4D-DRAMA with each of the other two methods.

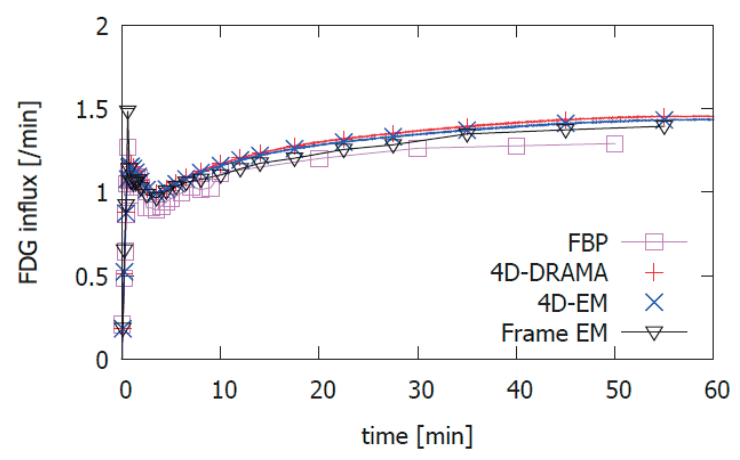

Fig. 7 Comparison of tTACs in the ROI averaged from 3 mice, reconstructed using the three methods. Plot of scanner-bundled FBP is shown as reference.

sinogram-based FBP method bundled with the scanner.

Parametric images indicating FDG-influx $K_{i}$ were estimated from the reconstructed tTACs. Figure 8 shows typical cardiac parametric images. Table 2 summarizes the mean and SD of the parametric images within the ROI, and $p$ values obtained from paired t-tests comparing the voxel values in the ROI from 4D-DRAMA and those from each conventional method. No significant differences were observed $(p>0.05)$. The image estimated from the curves reconstructed using 4D-DRAMA presented less variance than those reconstructed using the two conventional methods. Figure 9 shows the horizontal profile of the line drawn on Fig. 8.

\section{Discussion}

In this paper, a fast algorithm is proposed and its performance is reported. The reconstructed tTACs in Figs. 5-7 indicate that the proposed method; 4D-DRAMA, worked sufficiently well with real PET data, requiring only half the number of iterations of the conventional algorithms. The computational times were shortened by 17 h. As shown in Figs. 5 and 6, no differences were observed between the tTACs reconstructed using 4DDRAMA and those using 4D-MLEM. We found a slight difference (particularly around the early phase) between the tTACs obtained using 4D-DRAMA and those using frame-by-frame EM. This could arise from the use of basis functions that allow estimation to take into account the

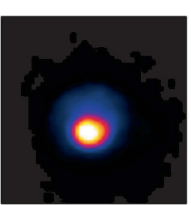

(a) 4D-DRAMA

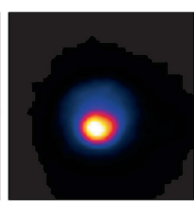

(b) 4D-EM

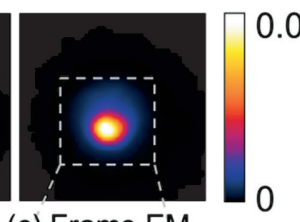

(c) Frame EM

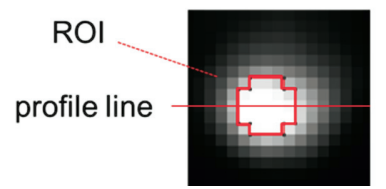

Fig. 8 Resulting ${ }^{18} \mathrm{~F}$-FDG influx images obtained at one slice. The enclosed area shown in the bottom image depicts an ROI, and the horizontal line depicts the position of the profiles shown in Fig. 9.

temporal correlation within each tTAC.

Tissue TACs generally show gentle changes, whereas the curve could have steep changes depending on the tracer materials and other factors. In the case of steep changes, the proposed method may fail to fit the basis function model, which could generate more error in the parametric images. Thus, we need to make a careful decision to choose the basis functions.

The computational speed is mainly determined by the number of iterations. The processing time of the forward and back projections depends on the number of list-mode data and forms the main bulk of the total computation. Moreover, such calculation depends on the number of list-mode events. Indeed, in Mouse 1, for instance, the computational time per iteration (without pre-computation) was similar in all three methods.

Figure 8 compares the FDG influx images from the tTACs estimated using the three methods. As shown in Table 2, 4D-DRAMA had smaller SD in the ROI than conventional methods have, in Mouse 1 and Mouse 3. In Mouse 2, 4D-DRAMA showed the worst SD. We chose the same number of subsets for the three mice, although the optimal number of subsets could vary depending on the data (such as total number of events). This might have worsened the result slightly because the data of Mouse 2 had the lowest count of the three. Still, no significant differences were observed between 4D-DRAMA and the other two methods in all the mice. We speculate that the 

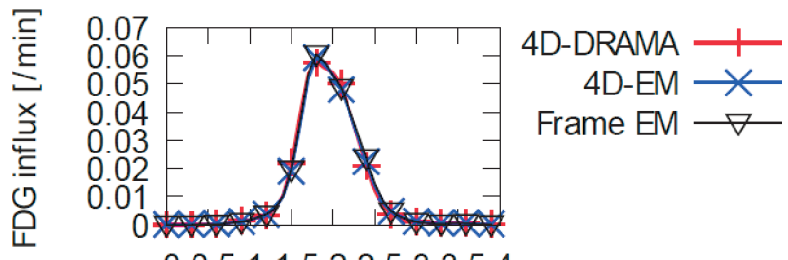

00.511 .522 .533 .54

distance $[\mathrm{cm}]$

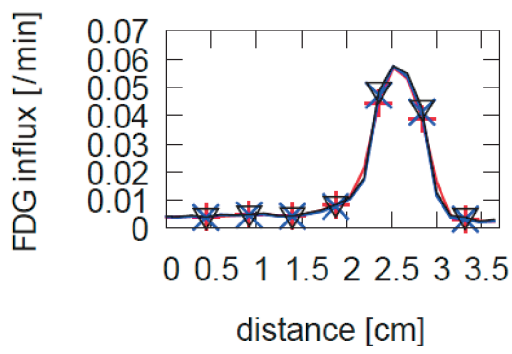

Fig. 9 A horizontal profile (top) and an axial profile (bottom) of the influx images in Fig. 8.

number of events might affect the influx value, which result in the differences in mean values among the three mice. In Fig. 9, we plotted the horizontal profile through the line on the ideal image to examine the differences among the influx images of Mouse 1 . The line of $4 \mathrm{D}$ DRAMA had a slightly lower peak than those of conventional methods, but there was only very slight difference in the curve.

The results indicate that 4D-DRAMA is capable of producing estimations with the same quality as conventional methods. These results agree with the result of a previous simulation study [7].

In this study, we only evaluated the cardiac region and the result might include the partial volume effect. At the next stage, we should evaluate regions with less errors, such as the brain.

The proposed method still requires empirical settings such as the definition of the relaxation parameter constants and the number of subsets (which is related to pre-computational time). The determination of these settings could be a subject for future study.

\section{Conclusion}

In this study, we validated a spatio-temporal reconstruction algorithm based on DRAMA using ${ }^{18} \mathrm{~F}$-FDG mouse dynamic PET data. We compared the proposed method with two conventional methods. The proposed method produced images within the cardiac region of mice with similar variances as the conventional methods but requiring shorter computational time. These results indicate that the proposed method can produce images with sufficient quality but remarkably shorter computational time than current conventional methods.

\section{References}

1. Watabe H, Ikoma Y, Kimura Y, Naganawa M, Shidahara M: PET kinetic analysis- compartmental model. Ann Nucl Med. 20(9), pp. 583-588, 2006.

2. Patlak CS, Blasberg RG: Graphical evaluation of blood-to- brain transfer constants from multiple-time uptake data. Generalizations. J Cereb Blood Flow Metab. 5 (4), pp. 584-590, 1985.

3. Nichols TE, Qi J, Asma E, Leahy RM:Spatiotemporal reconstruction of list-mode PET data. IEEE Trans Med Imag. MI-21 (4), pp. 396-404, 2002.

4. Reader AJ, Sureau FC, Comtat C, Trébossen R, Buvat I: Joint estimation of dynamic PET images and temporal basis functions using fully 4D ML-EM. Phys Med Biol. 51, pp. 5455-5474, 2006.

5. Browne J, De Pierro AB: A row-action alternative to the EM algorithm for maximizing likelihood in emission tomography. IEEE Trans Med Imag. MI-15 (5), pp. 687-699, 1996.

6. Tanaka E, Kudo H: Subset-dependent relaxation in blockiterative algorithms for image reconstruction in emission tomography. Phys Med Biol. 48(10), pp. 1405-1422, 2003.

7. Nakayama T, Kudo H: Derivation and implementation of ordered-subsets algorithms for list-mode PET data. Proc of 2005 IEEE Nuclear Science Symposium, Vol. 5, Puerto Rico, pp. 1950-1954, 2005.

8. Kon T, Obi T, Tashima H, Ohyama N:A proposal of spatio-temporal reconstruction method based on DRAMA. IEICE Trans Inf Syst. E96-D(4), pp. 819-825, 2013.

9. Kon T, Obi T, Tashima H, Yamaguchi M, Ohyama N: A modification of spatio-temporal reconstruction method based on DRAMA with Time dependent subsets. Proc of International Forum on Medical Imaging in Asia 2011, Okinawa, pp. 134-137, 2011.

10. Hashizume N, Kimura Y, Seki C, Nishimoto T, Kitamura K, Kanno I: Development of micro-liter order blood counting system for small animal molecular imaging using PET - validation with mouse study-. Proc of 2011 World Molecular Imaging Congress, San Diego, T213, 2011.

\section{Tatsuya Kon}

Tatsuya Kon received the B.E. degree in computer science and the M.E. degree in information processing from Tokyo Institute of Technology, Tokyo, Japan, in 2009 and 2011, respectively. He has been a doctoral course student in information processing at

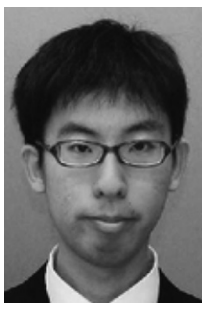
Tokyo Institute of Technology since 2011. His research interests focus on medical image reconstruction for PET.

\section{Takashi OвI}

Takashi OBI received the M.E. and Ph.D. degrees in information processing from Tokyo Institute of Technology, Tokyo, Japan, in 1992 and 1997, respectively. He was a Research Associate (1995-2002) at Imaging Science and Engineering Lab., Tokyo

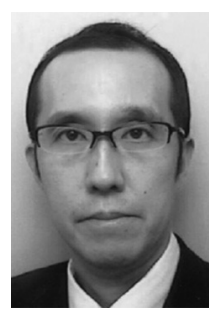
Institute of Technology and a Visiting Researcher (1998, 2000) at Univ. of Pennsylvania. He has been an Associate Professor at Tokyo Institute of Technology since 2002. His current interests include medical imaging, system security management and health informatics. 


\section{Muneyuki SAKata}

Muneyuki Sakata is a researcher of research team for neuroimaging, Tokyo Metropolitan Institute of Gerontology. He received his Ph.D. degree in 2005 from Nara Institute of Science and Technology. His major research interests focus on data analysis of

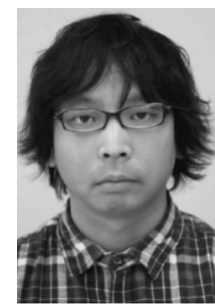
neuroimaging.

\section{Nagaaki OHYAMA}

Nagaaki OнyамA received the Ph.D. degree in information processing from Tokyo Institute of Technology, Tokyo, Japan, in 1982. He was a Research Associate (1983-1988) and an Associate Professor (1988-1993) at Imaging Science and Engineering Lab.,

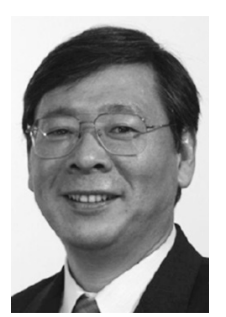
Tokyo Institute of Technology and a Visiting Researcher (1986-1989) at Univ. of Arizona. He has been a Professor at Tokyo Institute of Technology since 1993. His current interests include medical imaging, e-government and procurement systems.

\section{Yuichi KIMURA}

Yuichi KImURA graduated from Graduate School of Science and Engineering, Waseda University, in 1984, Tokyo, Japan and received the $\mathrm{Ph} . \mathrm{D}$. degree from Waseda University in 1991 and the D. of Medical Science degree from the Tokyo Medical and Dental University, Tokyo, Japan, in 2001.

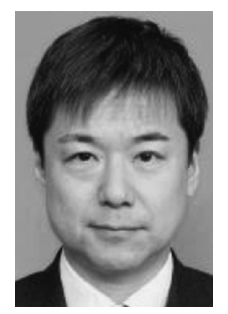

He served as Assistant Professor of Waseda University, Tokyo, Japan (1988-1991), Nihon University, Tokyo, Japan (1991-1994), Assistant and then Associate Professor in Tokyo Medical and Dental University, Tokyo, Japan (1994-1999), Senior Research Scientist at Positron Medical Center, Tokyo Metropolitan Institute of Gerontology, Tokyo, Japan (1999-2007), and Team Leader, Image Analysis Team, and then Head, Standardization Section, Molecular Imaging Center, National Institute of Radiological Sciences, Chiba, Japan (2007-2013). Since 2013, he has been Professor, Department of Computational Systems Biology, Faculty of Biology-Oriented Science and Technology, Kinki University, Wakayama, Japan. His major field is data and image analysis of PET image in clinical situation, and he is interested in an application of statistical approach to clinical images in nuclear medicine and molecular imaging.

Dr. Kimura is Vice-president of Japanese Society for Medical and Biological Engineering, and a member of the Japanese Society of Nuclear Medicine, IEEE Engineering in Medicine and Biology Society, and other related societies. He received the awards from JSNM and JAMIT in 2000 and 1996. 